\section{Is leukotriene B4 one of the keloid marker? a fibroblast keloid study}

\author{
Yuli Kurniawati, ${ }^{1}$ Oki Suwarsa, ${ }^{2}$ \\ Achadiyani, ${ }^{2}$ Sudigdo Adi $^{2}$ \\ 1 Dermatology and Venereology \\ Department, Faculty of Medicine, \\ Sriwijaya University/RSUP Dr. Moh. \\ Hoesin Palembang; ${ }^{2}$ Postgraduate \\ School of Medicine, Padjadjaran \\ University, Bandung, Indonesia
}

\begin{abstract}
Keloid pathogenesis occurs due to the duration of prolonged inflammatory phase and increased production of various growth factors such as TGF- $\beta 1$ which may cause increasing fibroblast proliferation and collagen synthesis. Existence of one of the chemical mediators released during inflammation, leukotriene B4 (LTB4), in keloid pathogenesis specifically in the phases of inflammation and proliferation, is still unclear. The purpose of the study is to analyze the levels of LTB4 in keloid. Methods: Fibroblast culture that was done by explanting keloid and normal skin of a keloid patient. Measurement of LTB4 on keloid and normal fibroblast was done by Elisa method. This experiment was run in triplicate. Statistical test was conducted by t test for the unpaired data and Anova test. The experiment was done at cell culture laboratory of Medical Faculty of Padjadjaran University Bandung. Levels of LTB4 in keloid fibroblast was higher than that of normal skin fibroblast (mean 23143.27 vs $18191.85 \mathrm{pg} / \mathrm{ml} ; \mathrm{p}<0.05$ ). Conclusion, increased LTB4 levels in keloid fibroblast showed the existence of LTB4 role in the prolonged inflammatory process in keloid pathogenesis.
\end{abstract}

\section{Introduction}

A keloid is a scar tissue that is developed due to the abnormal response in the wound healing process. Moreover, a keloid is formed as a result of increasing fibroblast production and collagen synthesis, hence it being one of fibroproliferative disorders. ${ }^{1-3}$ Keloid incidents, in which estimated $15 \%$ to $20 \%$, accounts for having been suffered by Afro-Americans, Hispanics, and Asians. ${ }^{4,5}$ Keloid pathogenesis occurs as a result of longer inflammation and the increasing pro- duction of numerous growth factors such as Platelet Derived Growth Factor (PDGF), Transforming Growth Factor- $\beta$ (TGF- $\beta$ ), and Vascular Endothelial Growth Factor (VEGF). Transforming Growth Factor- $\beta$ (TGF- $\beta$ ) acts in fibroblast proliferation. One of the responses to the abnormal wound healing is the longer duration of inflammation phase. ${ }^{1,6}$ Due to the cell membrane damage, phospholipids as the cell membrane structure would release arachidonic acid, supported by phospholipase enzyme. During the inflammation phase, mediators of inflammation would be released, including the lipid mediator that is leukotriene (LTs)., ${ }^{1,7}$ Leukotriene B4 (LTB4) is a potent lipid mediator of inflammation. The main functions of LTB4 is to be leukocyte chemoattractant and to act in the inflammation pathogenesis, immune disorders, and fibrosis. ${ }^{7,8}$ An in vitro study shows that LTB4 is a chemoattractant for fibroblast and a regulator of fibroblast influx into the tissue. ${ }^{9,10}$ Leukotriene B4 could also be found on skin disorders such as atopic dermatitis, psoriasis, and systemic sclerosis. The existence of one of the chemical mediators released during inflammation, LTB4, in keloid pathogenesis specifically in the phases of inflammation and proliferation, is still unclear. ${ }^{7,8}$

\section{Materials and Methods}

The study was based on the approval of Ethic Commission of the Faculty of Medicine of Padjadjaran University in Bandung. There was only one patient whose keloid tissue to be taken. Keloid excision was done on the patient according to the standard operating procedure (SOP). The decision of the keloid diagnosis was taken by a dermatologist. As the tissue excision was completed, the next procedure being the preparation for the primary cell culture with skin explant method. The study utilized the popular medium for the fibroblast transport and growth, Dulbecco's Modified Eagle Medium (DMEM), shot with penicillin, streptomycin, and fetal bovine serum (FBS) or fetal calf serum (FCS). The study took place in the Cell Culture Research Laboratory of the Faculty of Medicine of Padjadjaran University/Hasan Sadikin Hospital in Bandung. The study materials consisted of the keloid fibroblast from the primary culture of keloid tissue and fibroblast culture of a patient's normal skin. The fibroblast culture applied in the study was the third subculture while the study objects included LTB4 ELISA kit. The inclusion
Correspondence: Yuli Kurniawati, Dermatology and Venereology Department, Faculty of Medicine Sriwijaya University/RSUP Dr. Moh. Hoesin Palembang, Indonesia

Tel.: +628127150511, +62711-61314172

E-mail: yk_indun@yahoo.co.id

Key words: fibroblast, keloid, leukotriene B4

Contributions: The authors contributed equally.

Conflict of interests: The authors have nothing to disclose.

Received for publication: 1 February 2019. Accepted for publication: 12 February 2019.

This work is licensed under a Creative Commons Attribution-NonCommercial 4.0 International License (CC BY-NC 4.0).

(C) Copyright Y. Kurniawati et al., 2019

Licensee PAGEPress, Italy

Dermatology Reports 2019; 11(s1):8045

doi:10.4081/dr.2019.8045

criteria of the study consist of patient's keloid which had never received any treatments and $>80 \%$ of cell viability on primary culture while the exclusion criteria was the cells grown on the culture, not on fibroblasts. Fibroblast culture that was done by explanting keloid and normal skin of a keloid patient. The measurement of LTB4 on keloid and normal fibroblast was done by Elisa method. This experiment was run in triplicate. The statistical test was conducted by $t$ test for the unpaired data and Anova test. The experiment was done at the cell culture laboratory of Medical Faculty of Padjadjaran University Bandung. The study utilized in vitro experimental study design with the comparative completely randomized design.

\section{Results}

The result of fibroblast cell culture showed keloid fibroblast grew more rapidly than normal skin fibroblast. Figure 1 showed keloid fibroblast started to grow from explant periphery while in normal skin, fibroblast grew on day 10. Figure 2 showed the growth of fibroblasts in keloid and Figure 3 showed normal skin reached $80 \%$ confluence, hence the subculture (Figure 4). Such result prompted the second stage of study. In this study, the LTB4 level in keloid fibroblast was higher than normal skin fibroblast, as seen on Table 1. 


\section{Discussion}

Cell culture has been commonly used and is a method required for studies of wound healing process. ${ }^{11,12}$ Fibroblast cell culture with skin explant method is a primary culture, produces fewer fibroblasts despite being more commonly used due to its better cell morphology and fewer risks on cell damage as a consequence from enzymatic process. Fibroblast has an important role in the wound healing process. One of the abnormal wound healing processes could induce tissue fibrosis similar to that in keloid. ${ }^{13}$ Keloid occurs due to the abnormal wound healing process comprising elonga-

Table 1. The Comparison of LTB4 Levels in Keloid and Normal Skin Fibroblasts.

Variable Normal Keloid $\underset{P}{\mathrm{P}}$

\begin{tabular}{lc} 
LTB4 $(\mathrm{pg} / \mathrm{mL})$ & 0.01 \\
Mean $\quad 18191.85$ & 23143.27 \\
\hline Std. Deviation 1176.83 & 1782.15 \\
Std. Error mean679.44 & 1028.92 \\
\hline \multicolumn{2}{l}{ Note : *based on unpaired t-test $(\mathrm{p}<0.05)}$.
\end{tabular}

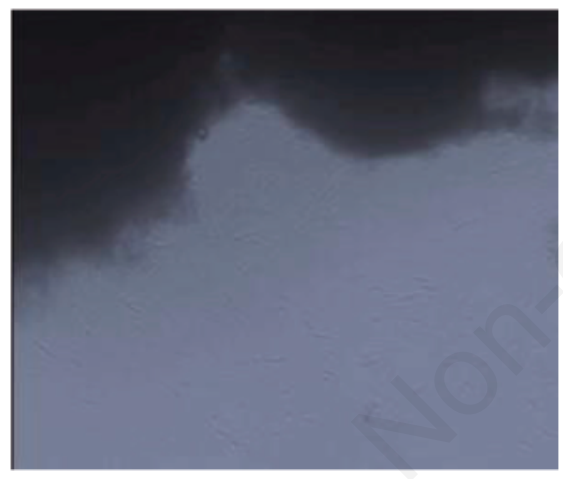

Figure 1. Keloid fibroblasts on day 8.

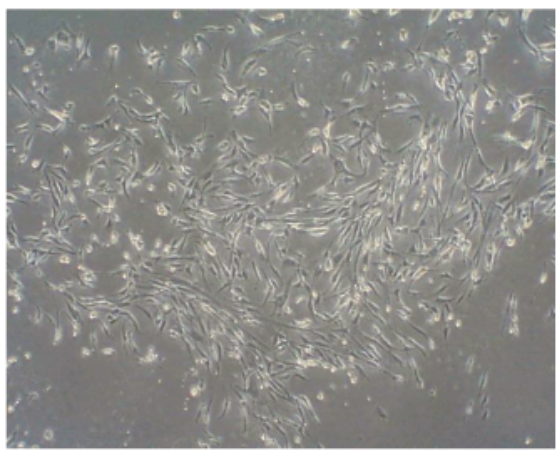

Figure 3. Normal skin fibroblasts on day 14. tion in proliferative phase and delayed remodeling. In addition, a theory has stated that keloid occurs due to the prolonged inflammatory phase. ${ }^{1,13}$ Leukotriene is one of the arachidonic acid metabolites, which is released through 5-lipoxygenase pathway (5-LO) ${ }^{7,9}$ Leukocytes are the main source of leukotriene. However, other cells such as macrophages, monocytes, fibroblasts, epithelial cells, and platelets are also able to produce leukotrienes. Leukotriene B4 is a potent lipid mediator released by the activation of neutrophils, macrophages, and mast cells; as well as a chemoattractant that is a fibroblast chemotactic factor. ${ }^{14}$ In prolonged inflammation, such cells would stay throughout the wound healing process with the total amount of macrophages and leukocytes higher in keloid fibroblast than those in normal fibroblast. ${ }^{14}$ The level of leukotriene B4 increases in several skin disorders such as atopic dermatitis, psoriasis, and systemic sclerosis though based on the researcher's knowledge, there has been no prior studies on the LTB4 level in keloid. In the study, the level of LTB4 in keloid fibroblast was significantly higher than normal skin fibroblast with the significant value (2-tailed) of LTB4 0.01 less than the $p$ value $<0.05$ as seen on Table 1 . The study result is in tune with a theory stating that keloid pathogenesis occurs as a conse-

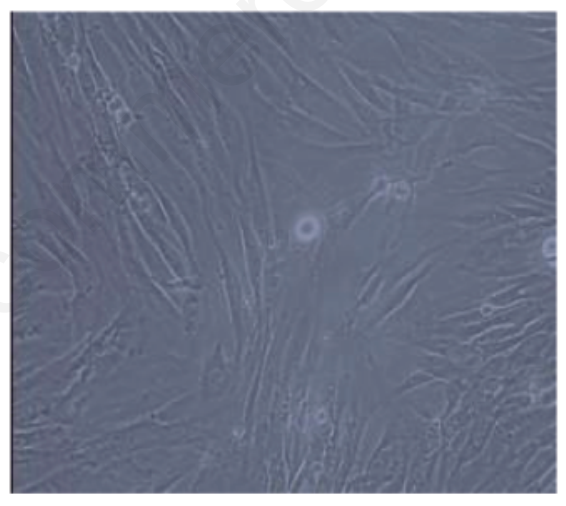

Figure 2. Keloid fibroblast day 14.

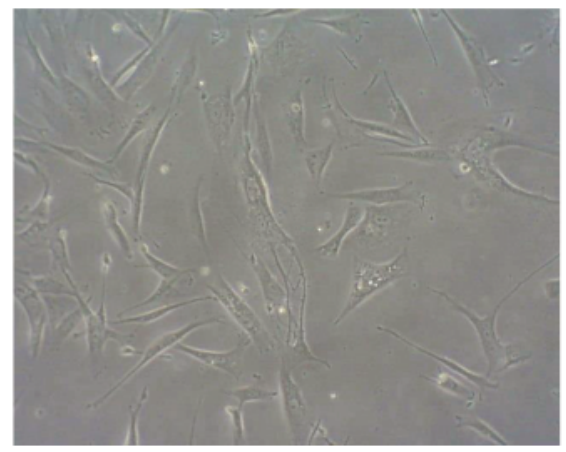

Figure 4. Keloid fibroblasts in subculture 1. quence of prolonged duration of inflammation phase. ${ }^{15}$ In a chronic inflammation, cells involved in the mechanism of inflammation would remain, including neutrophil, therefore the increase of LTB4 level could also be found in chronic inflammation. ${ }^{14,15}$ Further research is required to explore the role of LTB4 in keloid pathogenesis. The limitations in this study consisted of the sample which was being taken from the patient's keloid by elliptical excision; there has been no clear macroscopic boundary to differ normal skin from keloid, therefore during the excision, there was an open possibility for a part of keloid to have been carried into the normal tissue.

\section{Conclusions}

Increased LTB4 levels in keloid fibroblast showed the existence of LTB4 role in the prolonged inflammatory process in keloid pathogenesis. Further research with more sample is required to explore the role of LTB4 in keloid pathogenesis.

\section{References}

1. Gauglitz GG, Hans C Korting, Tatiana Pavicic, Thomas Ruzicka, Marc GJ. Hypertrophic scar and keloids: pathomechanisms and curret and emerging treatment strategies. Mol Med.2011;17:113-25.

2. Huang C, Ogawa R. Roles of lipid metabolism in keloid development. Lipid in Health and Disease. 2013: 1260.

3. Chenyu H, Ogawa R. Fibroproliferative disorders and their mechanobiology. Connective Tissue research. 2012; 53(3): 187-96.

4. Bran GM., Goessler UR., Hormann K., Riedel F, Sadick H.. Keloids: Current concepts of pathogenesis (Review). International Journal of Molecular Medicine. 2009 (24): 283-93.

5. Wojciech Bienias, Andrzej Kaszuba. Keloids: current prophylactic and therapeutic methods. Postep Derm Alergol.2012;XXIX,6:446-50.

6. Stephan Scheml, Rolf Markus S, Lukas Prantl, Michael Landthaler, Philipp Babilas. Wound healing in the 21st century. J Am Acad Dermatol. 2010; 63:866-81.

7. Sharma JN, Mohammed LA. The Role of leukotrienes in the pathophysiology of inflammatory disorders: Is there a case for revisiting leukotriene as therapeutic targets?. Inflammopharma- 
cology. 2006;14:10-16.

8. Takehiro Izumo, Mitsuko Kondo, Naoki Arai and Atsushi Nagai. Role of Leukotriene B4 reseptor antagonist in pulmonal fibrosis. AoRM.2010;1-5

9. Wen-Hsiang Su, Ming HC, Wen LL, Tsung ST, Wen HC, Chien SC, Peng HW. Non steroid anti inflammatory drugs for wounds: pain relief or excessive scar formation?. Mediator of Inflammation. Vol 2010, article ID 413238, 8 pages

10. Mathis S, Venkatakrishna RJ, Boddulur IH. Role of leukotriene B4 receptors in rheumatoid arthritis. Autoimun Rev. 2007; 7(1): 12-17.
11. Jie Li, Zuleika LBM, Robert SK. Wound Healing. In Robinson JK, William H, Daniel MS, Alina F, eds. Surgery of the skin, prosedural dermatology. 2nd ed. Mosby Elsevier.2010: 95-109.

12. Gregory CS, Jeffrey MD. Repair, Regeneration and Fibrosis in Raphael R, David SS eds. Rubin's Pathology:clinicopathologic Foundations of Medicine. 6th ed. Lippincot Williams Wilkins.Philadelphia. 2012:83-113.

13. Ahmad S, Azadeh E,Imam S, Thirumulu PK. Keloid scarring: understanding the genetic basic, advances, and prospects. Archive of Plastic Surgery. 2012;39:184-9.

14. Toshihiko Hayashi, Jun Nishihira, Yoshikazu Koyama, Satoru Sasaki and Yuhei Yamamoto. Decreased Prostaglandin E2 production by inflammatory cytokine and lower expression of EP2 reseptor result in increased collagen synthesis in keloid fibroblas. Journal of Investigative Dermatology. 2006;126:990-7.

15. Graziano R, Carmine DI, Pio C, Theoharis CT, Nicolantonio DO. Advances in therapy with antileukotriene drugs. Annal of clinical \& Laboratory Science 2004: 34:4: 379- 